\title{
RÉFLEXION SUR UN SYSTÈME GÉNÉRATIF-TRANSFORMATIONNEL DU COMPORTEMENT POUR LA COMPÉTENCE PRAGMATIQUE DU CECR
}

\author{
Rudy Kohwer \\ Edvania Gomes da Silva*
}

\begin{abstract}
RÉSUMÉ: Nous confions initialement notre objectif à la psychologie mentaliste. Ceci pour le regard critique que Chomsky (1969) apporte sur les idées de la psychologie comportementaliste, vue par Skinner (1957). Si la philosophie moniste de ce dernier conçoit l'organisation de l'environnement comme cause de la modification du comportement, nous apportons notre propre définition des actes de parole, afin d'étudier l'autre tenant scientifique de ladite modification, ou l'objet suivant : L'agir par un état de conscience mêlant les expériences déjà vécues à l'expérience nouvelle du présent. Les deux nouvelles dimensions du CECR (2000), et notamment l'approche novatrice dite actionnelle sous-tendue par la compétence pragmatique, laquelle fonde le socle théoricométhodologique de cette recherche, enveloppe notre contexte pédagogico-didactique, un manuel didactique comme corpus de référence. En conséquence, la méthode pragmatique et la théorie génétique de la vérité, conçue par James $(1911$; 1913), et ce, par le renforcement de la doctrine physique de Bacon (1851) et celle intellectualiste de Bergson (1965), ou les progrès qu'il apporte en matière de conséquence sur la mémoire, vérifient l'étude de notre objet, en termes reformulés, l'action de l'organisme en contexte naturel et pédagogique pour l'apprentissage du français comme langue étrangère. Nos considérations sont d'ordre prospective en le sens que, cette réflexion, pour un système générative-transformationnel du comportement verbal, s'inscrit dans l'actualité et pour l'évolution d'un tel enseignement en matière de psychologie.
\end{abstract}

MOTS-CLÉS: Comportement verbal; Linguistique des langues étrangères; Pragmatisme

\section{Introduction}

Le Conseil de l'Europe (2000, p. 15) publie le nouveau Cadre européen commun de référence pour les langues (CECR). Celui devient novateur en le sens que, « si les actes de parole se réalisent dans des activités langagières, celles-ci s'inscrivent elles-mêmes à l'intérieur d'actions en contexte social qui seules leur donnent leur pleine signification ». En définitive, s'il soutient

\footnotetext{
* Mestre em Linguística Aplicada pela Universidade Federal da Grande Dourados (UFGD). Doutorando em Memória: Linguagem e sociedade pela Universidade Estadual do Sudoeste da Bahia (Uesb). Bolsista CAPES (Coordenação de Aperfeiçoamento de Pessoal de Nível Superior).

** Doutora em Linguística pela Universidade Estadual de Campinas (Unicamp). Professora Titular da Universidade Estadual do Sudoeste da Bahia (Uesb). Realizou estágio pós-doutoral em Linguística na Unicamp.
} 
deux dimensions, a) un mode d'apprentissage des langues par une perspective de type actionnel sur b) les bases d'une didactique du plurilinguisme et de l'interculturalité, nous définissons comme suit le mode de fonctionnement de sa nouvelle perspective : Agir dans le contexte socio-culturel naturel où se produisent les habitudes et les manières de se comporter verbalement, elles-mêmes reproduites dans le contexte pédagogique concret (la salle de cours) et dans la ressemblance. En conséquence, une telle action créée l'agir par un état de conscience mêlant les expériences déjà vécues à l'expérience nouvelle du présent, notre objet d'étude.

Nos comportements, entourés de normes verbales, établissent nos actions avec éducation, ou bonne conduite sociale. Cette action est notre définition des actes de parole concernés pour l'acquisition de la compétence à communiquer et la signification des activités dans la méthode d'apprentissage du français comme langue étrangère, encadrée par le CECR (2000). Notre conviction, évaluer notre définition, noue deux directions. La première, tournée vers l'avenir, considère les conclusions à la suite de l'initiative du gouvernement Fédéral Helvétique, c'est-à-dire du Symposium Intergouvernemental qu'il organisa en 1991. Si les apports des auteurs, du groupe de travail et de projet, permirent la restructuration du CECR de 1993 à 1996 et à partir de l'approche communicative traditionnelle, puis son approbation en 1997 et enfin sa publication en 2000, les méthodes de français concernées furent repensées vers une approche dite actionnelle. Le manuel didactique LATITUDES. 1 : Méthode de français (2008), des auteurs Régine Mérieux \& Yves Loiseau, présente notre corpus de référence associé à son manuel pédagogique LATITUDES. 1 : Méthode de français. Guide pédagogique (2008).

La deuxième direction, tournée vers le passé, considère les contributions des théoriciens et praticiens engagés sur l'évolution de la didactique des langues étrangères, tels que Canale \& Swain (1980), Dumortier (2007), Moirand (1982), Bérard (1991) et Chomsky (1969). Sous l'horizon de la psychologie mentaliste pour une compétence d'ordre linguistique, ce dernier linguiste pose un regard critique sur les tenants de la thèse du conditionnement opérant de Skinner (1957). En contrat avec le courant fonctionnaliste américain, celleci soutient, par une psychologie scientifique de type béhavioriste (comportementaliste), les nouvelles méthodes audio-visuelles appliquées aux manuels didactiques encadrés par le CECR (2000). Entre les conceptions scientifiques de ces deux chercheurs, nous présentons le dilemme suivant : Soit la cause convient en l'organisation de l'environnement, comme le conçoit la philosophie moniste de Skinner (1957), alors l'effet est la réponse opérante du comportement et reliée à son renforcement, soit elle est l'état de conscience du comportement et l'effet devient le milieu. En conséquence, nous centrons nos recherches sur une 
action dans l'introspection, pour tourner ce dilemme en une hypothèse où l'agir de l'organisme serait la cause de l'organisation de son environnement.

Enfin, les autres auteurs proposent des recherches et leurs discussions par des articles déjà dirigés vers l'autre tenant du fonctionnalisme, le paradigme de la pragmatique. Pour être le socle prospectif des fondements théorico-méthodologiques des nouvelles méthodes d'apprentissage, celui-ci est en ce sens notre encadrement pour la mesure et l'évaluation des résultats, alors sur la base d'une réalité soutenue par James (1913) et propre à une théorie générale de la vérité. Et, les analyses débuteront sous l'angle de la doctrine physique de Bacon (1851), laquelle renforcera cette réalité : Celle-ci soutient que les faits en relation possèdent une qualité, en somme, leur condition d'exister et déterminée par un fait plus général, lorsque ce dernier est la transformation des faits particuliers en relation. Enfin, la doctrine de James, l'empirisme radicale ou l'expérience pure, soutient une méthode d'observation et d'expérimentation fondée sur les relations entre les faits particuliers, et leurs conséquences en contexte empirique. Nous y soumettrons alors l'action naissante selon Bergson (1965). Ainsi et pour achever, nous aboutirons à l'interprétation de la conséquence pratique, soutenue par James (1911).

En définitive, si notre objectif devient la proposition d'une ressource stratégique, lesdits didacticiens l'épaulent en reconsidérant, spécifiquement et préalablement aux discussions sur le nouveau paradigme, les ressources stratégiques appliquées sur les diverses composantes pour l'acquisition de la compétence à communiquer. Si leurs recherches prétendent se fonder sur le compromis espace-temps, celles-ci concernent l'application des stratégies de compensation lors de l'acquisition de la compétence à communiquer. La concernant, Canale et Swain (1980 apud BÉRARD, 1991, p. 19) donnent leurs catégories. Exerçant un pouvoir de compensation sur la composante grammaticale et sociolinguistique, la composante stratégique est donc concernée dès le début de l'apprentissage et remet à « l'ensemble des stratégies de communication qui permettent de compenser les ratés de la communication ». En revanche et pour Moirand (1982, p. 20), les ressources sont aussi de compensation, mais seulement au moment de l'actualisation de ladite compétence ou quand se présente les quatre composantes - linguistique, discursive, référentielle, socioculturelle - à l'apprentissage, alors surgissent les «stratégies individuelles de communication ».

Nous pouvons conclure qu'avant la réorganisation du CECR (2000), l'intérêt portait sur l'approche dite communicative, mais vers son évolution lorsque, le problème de la primauté sur la relation entre les composantes était et est encore le centre des débats. Ainsi, la composante référentielle soupçonne, tout autant que la notion d'autonomie puisque l'organisme agit 
dans ses contextes naturels selon la nouvelle approche nommée ci-dessous, une avancée en faveur de la restauration dudit document officiel. Bien qu'il ne se soit pas passé de ladite approche traditionnelle, cependant, il lui greffera l'approche dite actionnelle, et ce, de façon légitime face à un monde en constante évolution vers sa globalisation et son autonomie, en somme, ce que nous inspire la composante référentielle de Moirand. Mentionnée par Bérard (1991, p. 19), celle-ci possède un grand attrait pour notre recherche sur le défi que l'on s'est donné, une ressource stratégique par l'action sociale qui considère « la connaissance des domaines d'expériences et des objets du monde et de leur relation ».

\section{Deux faits particuliers pour une expérience : Vers un besoin d'état de conscience}

Nous mettons à contribution l'action qui provient, avec le mot pratique, de l'acception

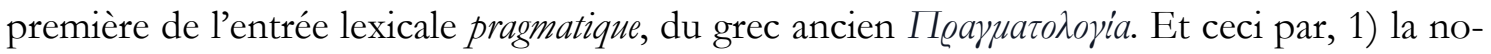
tion d'autonomie chère aux ressources stratégiques et 2) l'expérience à concevoir en conformité avec un apprentissage compris dans une durée sans intervalle d'interruption, en le sens du dispositif de la perspective actionnelle définie en introduction. Qu'il puisse en être ainsi, c'est dire de ces deux notions qu'elles sont définitoires de la pleine signification des activités de compréhension orale globale et de réception orale. Celles-ci, introduites par trois actes de parole sous chaque unité et en assurant par un support audio-visuel, ou soit audio ou soit visuel, la contextualisation de chacun d'eux, permettent ensuite le réemploi des normes sociales de langage et au moyen des contenus de production orale ou écrite, ou bien les deux, desquels débouche une tâche finale en vue de conclure l'unité, et ce, par un exercice d'écriture et/ou d’oralité en contexte social réel, et déterminé par la méthode didactique.

Cette chronologie étant posée, de l'ordre de la didactique, nous remarquons naturellement une répétition des normes sociales, lesquelles se succèdent au fil des contenus. Pour autant, nous souhaitons mettre à la lumière le processus de modification du comportement qui s'y prête. Considéré comme étant une technologie en les termes du conditionnement opérant skinnérien, ce processus prouve ainsi la scientificité de sa méthode d'expérimentation. Dans les soubassements théoriques des nouvelles méthodes audio-visuelles en didactique des langues, depuis les cinq dernières décennies du XX $\mathrm{XX}^{\mathrm{e}}$ siècle, si l'on agit sur un environnement conditionneur, l'organisation d'un certain contexte, notre action peut être fonctionnellement reliée en direction de compensations, renforcements ou conséquences, c'està-dire d'une récompense qui représente la réussite même de la consigne réalisée. Dans un contexte scientifique ou de laboratoire, cette satisfaction correspond aux résultats des 
analyses, pour ce qui est des travaux expérimentaux sur la vérifiabilité des théories de type stimulus-réponse-renforcement.

Toutefois, les seules contributions de cette psychologie scientifique données aux organes sensori-sensoriels (les sens), sont de l'ordre d'une philosophie moniste qui se dénoue a) des considérations pour la structure de l'organisme et b) de la perception interne. Par conséquent, notre objet d'étude est la face opposée de cette philosophie, lorsque la ressemblance, pour la relation des faits ou fragments d'expériences, est perçue et contenue en des moments et lieux différents sur l'échelle de temps, comprise dans toute la durée de l'apprentissage et remplie par la présence de l'organisme dans et hors contexte pédagogique. Dans une certaine mesure et en notre faveur, cette psychologie comportementaliste, étant une variante des psychologies appliquées, peut créer un amalgame pour les autres monistes, ceux de l'introspection. En effet, Chomsky (1969, p. 31) l'indique en citant que « Olds a rapporté un cas de renforcement par stimulation directe du cerveau, dont il a conclu que la récompense ne satisfait pas nécessairement un besoin physiologique, et ne supprime pas nécessairement un stimulus de besoin ». Ce stimulus serait, pour nous, l'attention pour un état de conscience efficient.

En quelque sorte et pour nos analyses, cette psychologie, acclimatons-la, cherchons pour l'expérimenter, à la faire se situer dans un contexte philosophique empirique. Le besoin serait un état de conscience adapté à nos actions dans ladite détermination de durée, lorsque Skinner (1957) définit, signalé par Chomsky (1969, p. 19), qu'« une autre variable qui peut affecter le taux [de réponse] est le besoin, que Skinner définit opérationnellement en termes d'heures de déprivation de [renforcement ou compensation] et du conditionnement sur la force de la réponse ». Le test est alors le besoin adapté par les actions de l'organisme en contexte naturel. Sinon, laissons cette psychologie en ce que son auteur la conçoit, à savoir et selon son paradigme expérimental du conditionnement opérant : La force de la réponse et le renforcement qui la contrôle sont l'effet d'une cause leur étant contingente par le besoin (la déprivation). La cause, étant l'organisation d'un environnement de laboratoire, réalise alors les conditions propices au stimulus de nature physiologique (sensation auditive ou visuelle). Or et quant à nos propres conceptions du besoin, la structure de l'organisme est la cause qui organise son milieu naturel. Nous expliquons brièvement notre ambition, comme notre entendement cherche à la conceptualiser.

Prenons et jetons le besoin dans le contexte naturel, entre deux apprentissages en contexte concret. La structure de l'organisme, fonctionnellement en action et reliée à un état de conscience que l'attention concentre pour la volonté de récompense, remarque les 
ressemblances sur l'usage des normes sociales entre les deux sociétés, ici, les pronoms de traitement entre la langue maternelle et la langue d'apprentissage. Si c'est bien l'instant naissant d'une nouvelle expérience du présent dans l'intervalle, sa constitution tient en le passé, c'est-à-dire en les expériences connues, vécues, pour ainsi dire innées si dès le bas âge, l'éducation eût été de bonne foi. Qui plus est, c'est bien là lieu de modification du comportement verbal. Au passage, signalons que cette instant naissant dans l'intervalle est en ce qu'est l'intervalle chez Bergson (1965, p. 54), ainsi rédigée : «J'ai commencé par un état où je ne distinguais que ma perception ; je finis par un état ou je n'ai plus guère conscience que de mon automatisme : dans l'intervalle a pris place un état mixte, une perception soulignée par un automatisme naissant ». Nous y reviendrons ultérieurement aux analyses renforcées par la doctrine physique de Bacon (1851). Aussi, par un acte directif, nous insistons sur ce point fondamental : Estimer avec importance la ressemblance dans la différence et non dans l'identique proprement dit. Nous le démontrons maintenant.

Conclure en envisageant cette faculté qu'est l'attention et qui deviendrait une ressource stratégique dans le contexte pédagogique concret, est nécessairement l'effet corrélatif de deux variables, mieux disant, de deux faits particuliers pour valider l'expérience particulière qui s'en suit. Nous les posons sous formes de prémisses. Et, l'argument dans son entier aboutira à sa conclusion afin de rendre compte du passage du besoin physiologique vers un besoin d'état de conscience. Si pour un même pronom de traitement la norme de son usage ou d'utilisation du langage diffère - un faible degrés de qualité est suffisant - entre les deux sociétés, et si leur idiome respectif possède en leur utilisation naturelle, de bon droit civique, ceci de commun, c'est-à-dire l'existence et l'utilisation normal du traitement de la personne sous les conditions que le prochain paragraphe signalera, alors l'attention qui concentre un état de conscience particulier vers le besoin, est réalisable et productive pour la modification du comportement. En somme et dans notre cas, l'attention est une fonction particulière de l'organisme, justifiant alors que sa structure est fonctionnelle, et selon notre argument face à notre objet d'étude, réorganisable en même temps que se produit la génération ou la transformation d'un comportement.

Outre la conviction pédagogico-didactique susceptible d'accompagner le comportement dans sa conduite, grâce aux organes sensori-sensoriels selon la base théorique des nouvelles méthodes audio-visuelles, s'ils nous sont venus les pronoms de traitement en la pensée, sous l'acte de parole entrer en contact dans l'unité initiale du manuel didactique, cette révélation tient en l'envergure qu'a la bonne éducation représentée par les actes de civilité dans certains genres interactifs sociaux, tels que ceux requis dans le système hiérarchique des institutions 
de production de biens et services, de l'éducation, entre autres. La ressemblance des faits entre eux, et ceux dans l'action entre les deux contextes, cruciale pour la validité scientifique de la démonstration sous l'angle d'une expérience nouvelle et contingente avec les faits du passé, est concomitante avec la réorganisation de la structure fonctionnelle de l'organisme, et ce, de part l'action dans le présent de l'expérience nouvelle, laquelle résout sa nécessité à la volonté que l'homme à d'évoluer dans les sociétés modernes. Ainsi, du point de vue théorique et de la méthode, ou scientifique, ces faits confirment-ils l'expérience, en d'autres termes, la modification du comportement verbal, c'est-à-dire, est-ce que l'entrainement des pronoms de traitement, hors contexte concret d'apprentissage, peut avoir condition de renforcement pour le renforcement même, ou mieux, pour la conséquence pratique et de satisfaction tournée vers l'avenir, ou le succès des tâches à réaliser dans l'ensemble des contextes d'apprentissage des langues étrangères ?

Posons le point de vue didactique sur la méthodologie donnée par la composante fonctionnelle et reliée à la compétence pragmatique au regard du CECR (2000). Pour autant, débutons selon la définition que Dumortier (2007, p. 129) entend par compétence à communiquer: «l'aptitude à mobiliser les ressources nécessaires pour résoudre les problèmes inhérents à une situation de communication (au moins partiellement) nouvelle, apparentée à une classe de situations de communication familière à l'agent ». Si l'auteur parle en la logique de la perspective actionnelle comme l'inspire la seconde classe, et globalement ou pour les deux classes, la conclusion de notre argument valida notre ressource stratégique, et ce, par le besoin d'un état de conscience à prendre en contexte naturel. L'auteur initie de manière à ce qu'une question vienne à notre esprit, qui, de prime abord, nous suggère une théorie de la mémoire de type bergsonienne, plus spécifiquement, ses considérations sur la reconnaissance : S’il faut que le sujet apprenant, ou nous tous de façon généralisée, puisse entretenir les acquis produits dans son contexte naturel afin de les réutiliser à bon escient dans le contexte pédagogique, sous quelle forme l'action, après constitution de la nouvelle expérience ou modification du comportement, doit tenir dans la durée?

On parlerait volontiers d'habitude pour la résolution de ce problème de recherche. Néanmoins, ne voyons nous pas qu'un raisonnement sur la base d'un travail de répétitions identiques serait lassant, voir inefficient. Et pour cause, premièrement, le résultat qu'établit Bérard (1991, p. 13) avant la restructuration du CECR (2000), soit « certaines habitudes de l'élève dans sa langue maternelle, par exemple l'utilisation des règles grammaticales, ne sont pas réinvesties dans les méthodes audio-visuelles ». Outre qu'un réseau d'habitudes soit soutenu théoriquement par la méthode skinnérienne, nous pensons que les contenus rattachés 
aux contextes sociaux, dans ledit manuel didactique, sont en nombre insuffisant au regard de l'intégralité des habitudes dans la réalité. Deuxièmement, la réduction à néant d'une attitude curieuse et motivé, liée à la volonté de notre besoin d'état de conscience. Observons succinctement, comme promis, le point de vue didactique, ou mieux, quels sont les changements prévus pour attiser la curiosité en constance et de ce fait, comment entretenir les acquis selon la compétence pragmatique depuis la publication du nouveau CECR (2000) ?

Les répétitions, objet orale et écrit dans la succession des contenus sur les activités didactiques nommées par le premier paragraphe de cette partie, sont et selon notre ressource stratégique, efficaces lorsqu'associées à l'attention de l'organisme. En outre, cet état de conscience permet a) de réorganiser la structure fonctionnelle de l'organisme dans le contexte naturel, ainsi, b) de renforcer les répétitions par cette ressource stratégique, et ce, dans un mouvement de durée déterminée sans intervalle d'interruption, lequel étale les contenus d'apprentissage jusqu'à la tâche finale. Ainsi confondues, les deux piliers dimensionnels du CERC portent notre problématique. En les réitérant - la didactique du plurilinguisme et de l'interculturalité ; le mode d'apprentissage par la perspective de type actionnel, - nous posons les conceptions que revêt leur soutenance didactique : Reprenons par ce que cite initialement Dumortier (2007). Selon ledit document officiel (2000, p. 99), la composante fonctionnelle comprend l'aptitude à mobiliser les «schémas (modèles d'interaction sociale) qui [...] comprennent des suites structurées d'actions [...]. Sous leur forme la plus simple, on trouve des paires telles que $:$ question $\rightarrow$ réponse ; déclaration $\rightarrow$ accord/désaccord ; requête $\rightarrow$ acceptation/refus ; salutations/toast $\rightarrow$ réponse ».

Dans l'objectif de terminer la durée sur la tâche finale où sa réponse est notre besoin - en termes d'espace-temps, le besoin est l'action en ce qu'est l'intervalle entre deux présences du contexte pédagogique - en ce qu'est le besoin à des fins fonctionnelles dans les situations du domaine réel public, privée, éducationnel et professionnel, nous remarquons que ces quatre suites permettent de faire alterner différemment les répétitions, et ce, auxilié par une multitude de micro-fonctions préconisées dans le CECR (2000). Et nos observations sur ledit manuel didactique, notamment sur le mode de contextualisation de chaque acte de parole, nous font constater une évolution en faveur des méthodes audio-visuelles qui, cependant et pour être dénouées de la tradition ou des méthodes du structuralisme européen, se voient encadrées d'un fonctionnalisme américain, remarquablement bien représentée chez James $(1911$; 1913). Alors, si nous soutenons originairement notre objet d'étude par les conséquences des principes pour la théorie de la mémoire vue par Bergson, dans son essai intitulé Matière et mémoire : Essai sur la réflexion du corps à l'esprit (1965), surtout lorsque le philosophe 
parle d'utilisation de l'expérience du passé pour l'action présente sous forme de reconnaissance, et bien le tracé terminal de notre horizon s'étire par les considérations de la thèse de James (1913), à propos du pragmatisme dans ses oeuvres intitulées L'idée de vérité (1913) et Le pragmatisme (1911).

\section{Regard théorique sur les faits de l'expérience : la méthode philosophique et l'analyse}

L'intérêt scientifique du premier ouvrage prédomina pour le mouvement pragmatiste. De l'ordre de la théorie, James (1913, p. 9-10) y précise les fondements et les lois de sa théorie génétique de la vérité. Son optique, développer une thèse du pragmatisme sur les bases de « la 'valeur active' que doivent posséder les idées, pour être vraies, consiste en effets particuliers, physiques ou intellectuels, réels ou possibles, que ces idées peuvent déterminer de proche en proche à l'intérieur de l'expérience concrète ». En termes d'une philosophique plus moderne, l'auteur s'appuie sur sa propre approche méthodique ou pratique, en passant par la théorie de la réalité, explicitée dans son œuvre intitulée Le pragmatisme et publiée deux années auparavant. James (1911, p. 56-57) y définit que le côté pratique des expériences est abordé sur la conséquence des idées, en somme, en cherchant à interpréter chaque « conception d'après ses conséquences pratiques : que telle conception fût vraie, et non telle autre, quelle différence en résulterait-il pratiquement pour un homme? Qu'aucune différence pratique ne puisse être aperçue, on jugera que les deux alternatives reviennent au même ».

Alors qu'il reçut les honneurs d'Henri Bergson, qui rédigea son introduction, ce dernier nous dit les motifs du second ouvrage qui suivra deux années plus tard : «La structure de notre esprit est donc en grande partie notre œuvre [...]. Là est, ce me semble, la thèse la plus importante du pragmatisme, encore qu'elle n'ait pas explicitement été dégagée. C’est par là que le pragmatisme continu le kantisme » (JAMES, 1911, p. 13). Qu'en est-il de la modification du comportement verbal aux ordres de l'entendement de ce pragmatiste sur la propriété des idées, c'est-à-dire leur valeur active que nous résumons en une relation appuyée par le Nouvel Organum de Bacon (1851, p. 10), dans son ouvrage intitulée Oeuvres de Bacon. Par sa méthode, il porte notre vision que l'on a sur la réorganisation de la structure fonctionnelle de l'organisme. Pour l'auteur, il est question d'établir « des degrés de certitude, de donner de l'appui aux sens par une réduction des objets, mais en rejetant presque tout le produit des premières opérations de l'esprit qui suivent immédiatement les sensations, la route nouvelle $[\ldots]$ devant commencer aux perceptions des sens ».

En somme, nous trouvons intéressante l'analogie avec les sciences actives dans la mesure où la méthode de Bacon (1911) extrait, des faits particuliers, leurs principes et conduit 
à de nouveau faits particuliers, nous voulons dire en association avec la valeur active. Les considérations des rationalistes anti-pragmatistes, concevant les effets qui accompagnent la vérité sans cependant la constituer et qui plus est, l'existence nécessaire de l'objet quant à sa véridicité, sont pour James des conceptions trompeuses de la vérité, quand les idées peuvent ne pas être la copie fidèle d'un objet. Ces effets particuliers, nous les avons définis comme étant la corrélation de deux variables, relation que nous pouvons ramener aux qualités. En les réitérants - dans les deux sociétés et leur idiome respectif, la norme d'usage ou l'utilisation diffère pour un même pronom de traitement, et, leur utilisation naturelle a en commun l'existence et l'utilisation normale du traitement de la personne, - ces deux faits définissent l'expérience possible ou notre cause qu'est l'attention, laquelle concentre un état de conscience particulier vers notre besoin, lequel est la conséquence pratique où l'action dans l'intervalle espace-temps devient interprétable.

L'étude du sens qui concerne l'agir, a pour sa direction, cette propriété générale que sont les normes verbales de bonne conduite sociale. Les deux faits particuliers, mentionnés ci-dessus, qui en découlent, en modifient et limitent le sens, possèdent une série de principes. Pour le premier fait, disons-le, ceux-ci tiennent de conditions historiquement littéraires plus que culturelles, bien que sur la norme d'usage des pronoms de traitement tu e vòs, dans la société brésilienne, celles-ci tiennent en une virée plus culturelle. Son usage des normes était autrefois identique au français. Mais de nos jours, você marque l'empreinte culturel, vòs ayant été remis dans son contexte littéraire. Quant au pronom $t u$, son insistance sur les relations privilégiées, telles que la famille et les amis, ou sa marque d'usage, est bien plus forte dans la société brésilienne que française. Et quant au deuxième fait, les principes tiennent en de conditions historiquement sociales. Bref, nous n'irons point les mentionner une à une, la liste n'est que plus exhaustive sur les deux faits et l'intérêt est sans importance lorsque nous apercevons déjà des différences de ressemblance. Au delà des faits pris indépendamment où leurs principes respectifs déterminent leur forme, c'est-à-dire leur condition d'existence, la corrélation qu'ils entretiennent entre eux, par une série d'opérations interne et invisible, est notre cause, l'attention qui concentre un état de conscience.

On souhaite introduire ces principes dans notre besoin - l'action dans l'intervalle espace-temps - pour la transformation/génération du comportement et qui répondrait à notre objet d'étude : L'agir par un état de conscience mêlant les expériences déjà vécues à l'expérience nouvelle du présent. Nous avons mis à la lumière les principes, la forme des deux faits, en quelque sorte, l'objectif de la science humaine. Nous envisageons maintenant de mesurer et d'évaluer leur conséquence pratique sur l'effet, l'organisation de l'environnement, et valider 
notre ressource stratégique. Ou mieux, le premier point sur la détermination de la valeur active consistant en effets particuliers physiques, fut arboré par les deux prescriptions ou objectifs suivants de Bacon (1851, p. 90) : «au premier, la transformation des corps concrets d'une espèce en une autre espèce [...]; au second la découverte à faire dans toute génération et tout mouvement du progrès caché qui s'opère par une cause évidente, dans une matière connue jusqu'à ce que sa forme s'y trouve ». Il en va naturellement d'envisager maintenant la mesure et l'évaluation de la conséquence pratique de ces deux faits sur l'effet ou l'organisation de l'environnement, et tirer les considérations finales sur notre ressource stratégique.

Par conséquent, la détermination qui consiste en effets particuliers intellectuels selon James (1913), c'est Bergson qui soutient l'analyse de celle-ci. Sur ces deux mémoires et sur la perception des sens, quant à la première mémoire qui représente les souvenirs-images des événements de notre vie quotidienne,

\begin{abstract}
par elle deviendrait possible la reconnaissance intelligente, ou plutôt intellectuelle, d'une perception déjà éprouvée ; en elle nous nous réfugierions toutes les fois que nous remontons, pour y chercher une certaine image, la pente de notre vie passée. Mais toute perception se prolonge en action naissante ; et à mesure que les images, une fois perçues, se fixent et s'alignent dans cette mémoire, les mouvements qui les continuaient modifient l'organisme, créent dans le corps des dispositions nouvelles à agir. Ainsi se forme une expérience d'un tout autre ordre et qui se dépose dans le corps, une série de mécanismes tout montés, avec des réactions de plus en plus nombreuses et variées aux excitations extérieures, avec des répliques toutes prêtes à un nombre sans cesse croissant d'interpellations possibles. Nous prenons conscience de ces mécanismes au moment où ils entrent en jeu, et cette conscience de tout un passé d'efforts emmagasiné dans le présent est bien encore une mémoire, mais une mémoire profondément différente de la première, toujours tendue vers l'action, assise dans le présent et ne regardent que l'avenir. (BERGSON, 1965, p. 48)
\end{abstract}

Nous notons, lors d'un état de conscience d'attention pour effectuer l'agir, que la perception présente, dans le contexte pédagogique, vient chercher les représentations de l'expérience ou de ses fragments réalisés auparavant dans l'intervalle qu'est l'instant dans le contexte naturel. En les termes bergsoniens, la reconnaissance est attentive dans le sens ou la perception du passé, dans notre intervalle, vient à être pénétrée par notre perception du présent, afin qu'une ou des représentations en soient extraites dans un mouvement qui transforme alors la structure fonctionnelle de l'organisme.

Ainsi, il y a génération de la perception nouvelle du présent, et ce, par le comportement verbal écrit ou oral comme conséquence pratique. L’intervalle, la présence dans le contexte naturel et entre deux apprentissages dans le contexte pédagogique, fait naitre l'action qui se sert du passé pour son présent, en somme et là aussi en les termes bergsoniens, une action naissance. La tendance de celle-ci est vers l'avenir, dans notre cas, vers la future action 
présente dans le contexte pédagogique, après l'intervalle. En définitive et pour qu'un tel mouvement se fasse, nous avons souligné qu'il fallait une différence de qualité dans l'utilisation des pronoms de traitement, entre les normes d'usage dans les deux sociétés, et que cette différence de qualité devait se ressembler entre les deux sociétés. Or, avoir souligné cette nécessité fondamental devient, à ce stade, une hypothèse dont l'actuelle vérification va maintenant démontrer que sa nécessité est obsolète, et ce, par une variable de l'action, c'est-à-dire par l'habitude de répétition.

En effet, s'il est simple de comprendre que le déroulement d'une unité didactique, dans son entier, réalise plusieurs intervalles - présences dans le contexte naturel - et plusieurs présences dans le contexte pédagogique, il est alors censé que ces répétitions créent une habitude. Et en conséquence, soulignons au passage que Bergson (1965, p. 45) le soutient par son expérience vérifiant ces trois propositions quant aux « conséquences qui découleraient de nos principes pour la théorie de la mémoire », les représentations n'ont plus lieu d'intervenir dans l'action, c'est-à-dire dans la réalisation efficiente de la tâche finale, l'exercice d'écriture ou d'oralité. On pourrait donc dire, que cette tâche de la perspective actionnelle est l'action de la perception présente sans le passé ou la perception du passé. Mais alors, pour quelle raison y aurait-il encore perception ? Schématisons cette question à partir du commencement du mouvement, lequel prend la forme qui va suivre. Mais d'abord, voyons la formation d'une telle forme.

Sa constitution débute du souvenir posé sur une assise, en d'autres termes, sur une surface plane, matérialisée par le souvenir, la représentation. Il c'est donc crée un événement caractérisé par la formation d'une matière, avec ses contours, ses limites, ce que possède la représentation, comme une image. Or, cette surface plane, laquelle sert d'assise aux représentations, est sans quelconque extrémité, ni même dans sa largeur, sa longueur et sa hauteur, en somme, une aire ou un volume qui définit son espace comme un univers infini, bien que la naissance et la mort de l'organisme marquent respectivement son commencement et sa finalité, donc un certain périmètre. Mais justement et en ouvrant une parenthèse, là est toute la forme, lorsque des représentations se sont posées ici et là dans son espace, héritage des expériences de l'organisme. Fermons-la et continuons alors en parlant d'une substance sans consistance ni conscience, ne serait-ce que celle d'un air. Dans l'intervalle élargie, celle entre le début et la fin de l'organisme, nous avons ajouté que des représentations se sont posées.

La formation de cette forme ainsi définis, ensuite, un mouvement peut alors venir chercher cette matérialité et ce, par la perception du présent. À ce sujet, nous avons dit que la perception présente pénètre la perception passée ou la substance, afin d'y cueillir ses 
représentations, ainsi, réaliser la transformation du comportement. Nous avons aussi ajouté qu'il faut que l'état de conscience soit celui de l'attention, car il s'agit de connaissance, et tout particulièrement, de connaissances des faits de la réalité à propos des langues et leurs cultures. C'est ainsi que l'organisme a pris conscience, dans le contexte naturel, du premier de notre fait, c'est-à-dire de sa qualité. Si l'on apporte la première partie de la thèse intellectualiste de James (1913), notre qualité est en ce qu'est la qualité d'un sentiment ou d'une idée. Et celleci est vrai puisqu'elle est dans et la réalité, ce que fonde la théorie génétique de la vérité dudit pragmatiste. Enfin, si celui-ci parle de transcendance, par analogie, elle est un mouvement entre le sentiment, dans la substance de l'organisme, et sa qualité, dans la réalité. En le sens du pragmatiste ou pour que l'action puisse se faire, qualité du sentiment dans un contexte et le sentiment doivent se ressembler.

Ainsi, James (1913, p. 5) projeté vers la future expérience ou la conséquence pratique, revient sur l'importance de la ressemblance, lorsque la « qualité nouvellement créée ressemble à la qualité du sentiment q, je dirai que nous pouvons considérer le sentiment comme ayant connaissance de cette réalité ». Cependant et s'il revoit ainsi la vérifiabilité de notre hypothèse, c'est qu'il en est à l'étape de la série de principe que nous avons mentionnée auparavant, ceux qui ont vérifié la vérité de nos deux faits particuliers, vérité qui est le centre de sa thèse du pragmatisme. Dans un contexte d'étude de la didactique ou d'une perspective actionnelle qui remet à la réalité sociale, sa thèse suggère évidemment l'efficience de la réponse, c'est-à-dire la tâche finale réalisée. En revanche et par une pierre deux coups ou en revenant au contexte de la conscience et à l'habitude de la répétition, la perception présente ne va plus chercher les représentations, celles à l'intérieur de la perception du passé, mais les possède en son sein puisque l'action ou la perception présente est devenue mécanique, tel un automate, comme à la manière de réciter la leçon apprise en les termes bergsoniens, une sorte de mémoire ontologique.

\section{Conclusion}

Le besoin, selon Skinner (1957) et nos conceptions à ce propos, correspond à un espace-temps. Ce qui néanmoins nous oppose, est la définition de celui-ci. Respectivement, le besoin est une organisation de l'environnement de manière à ne disponibiliser aucune récompense, compensation ou conséquence à l'organisme expérimenté. En somme, cette organisation remet à une durée d'heures de déprivation où l'organisme est dans un environnement sans stimulus vers une réponse opérante. Cependant et dans notre cas, notre conception du besoin est la conséquence pratique, les faits de la réalité organisée par et dans la 
conscience de l'organisme qui, indépendamment de toute organisation du milieu, se disponibilise à n’importe quel moment de la durée, et ce, pour la réponse de la perceptive actionnelle ou la génération de l'exercice d'écriture ou d'oralité.

Cette disponibilité, nous l'avons encadré théorico méthodologiquement, est réalisée par un état de conscience d'attention. Au fond, si nous avons étudié et fait remarqué, par la voie de l'introspection, un autre mode d'adaptation du comportement, la raison en est les prescriptions de la perspective de type actionnel. En effet, alors que le comportement de l'organisme, dans le contexte naturel, se produit par les interactions quotidiennes, il est en ce sens évident que les nouvelles méthodes audio-visuelles, ou mieux, leurs supports tels que les illustrations et les audio du manuel didactique, n'encadrent pas les interactions spontanées, entre autres, entre membres de la famille, collèges de travail, etc. Qui plus est, la base de la pragmatique ou de cette vérité ici considérée, tient en l'organisme situé dans une réalité socio-culturelle, autour de faits réels qui valident les expériences.

\section{Considérations finales}

Notre ressource stratégique, appliquée dans l'intégralité de la durée, demanderait toutefois et pour les premiers apprentissages, une formation de l'organisme sur la fonction qu'a la perception du présent vers la perception du passé. Si nous tendons à une philosophie de l'esprit pour nos explications, il est vrai que pour démontrer l'application d'une telle fonction dans la pratique, nous avons indubitablement pensé à un soutient, la théorie générative ou transformationnelle chomskienne à propos de sa grammaire. Or, lorsqu'il s'agit d'un système de compétence linguistique rattaché à un acte créatif qui se veut générer les nouveaux énoncés sans quelconque soutient des expériences du passé, la théorie génétique du linguiste conçoit mal les fonctions que l'organisme peut mettre en place, lorsque soutenu par une théorie génétique de la vérité considérée par James (1913), laquelle se sert de réalités, de représentations échangées entre les individus et au cours de leur histoire.

Par conséquent et dans la continuité de l'apprentissage après une formation et les premiers enseignements, s'il nous est difficile de se confier aux structures mentales selon les conceptions chomskiennes, c'est que celles-ci et pour cet auteur, sont qualitativement différentes des réseaux d'habitude et des structures complexes du monde. Rappelons que notre entendement à nous, tient en une évolution vers la perception présente spontanée sans nécessité de la perception du passé. En revanche, nos conceptions ne peuvent pas signifier que les expériences ou fragments d'expérience retirés de la perception du présent, proviennent d'une structure innée sous l'angle chomskien ou dans le sens de la biologie génétique. On 
dirais plutôt qu'elle devient en quelque sorte innée par l'intermédiaire des répétitions et réseaux d'habitude, dans et par le fil conducteur du commencement des apprentissages jusqu'à leur finalité.

L'évolution de notre réflexion sur ces bases et pour un système générative-transformationnel du comportement, a cependant et, selon ce mouvement et cette intervalle, des variables à penser et définir en mesurant les conséquences, telles que l'inhibition dans les interactions sociales naturelles et les pertes d'attention liées à l'absence d'un enseignant dans le contexte naturel. Il conviendrait en ce sens de mieux cerner les enjeux du plurilinguisme et de l'interculturalité pour l'individu, ses besoins, ses attentes, ses projets pour l'avenir. Aussi et en termes scientifiques, l'apport d'une psychologie des sentiments pourrait renforcer nos conceptions théoriques, sans cependant dériver dans l'absolu, au risque de retirer la validité scientifique quant à l'évolution qu'aurait la recherche ici présente.

\section{REFLEXÃO SOBRE UM SISTEMA GERATIVO-TRANSFORMACIONAL DO COMPORTAMENTO PARA A COMPETÊNCIA PRAGMÁTICA DO CECR}

RESUMO: Confiamos inicialmente nosso objetivo à psicologia mentalista. Isso pelo olhar crítico que Chomsky (1969) desenvolve sobre as ideias da psicologia comportamental, do ponto de vista de Skinner (1957). Se a filosofia monista deste último concebe a organização do ambiente como sendo a causa da modificação do comportamento, propomos nossa própria definição de atos de fala, a fim de estudar uma outra perspectiva científica sobre a referida modificação, o que se configura no seguinte objeto: agir por um estado de consciência mesclando as experiências já vividas com a nova experiência do presente. Tanto as novas dimensões do CECR (2000) quanto a abordagem actionnelle, sustentada pela competência pragmática, a qual funda a base teóricometodológica dessa investigação, embasam o nosso contexto pedagógico-didático, que tem um manual didático como corpus de referência. Consequentemente, o método pragmático e a teoria genética da verdade, sob o ângulo de James (1911; 1913), e, com o reforço da doutrina física de Bacon (1851) e da doutrina intelectualista de Bergson (1965), ou o progresso que ele traz em termos de consequências da memória, conferem ao estudo do nosso objeto, em termos reformulados, a ação do organismo em um contexto natural e educacional para a aprendizagem do francês como língua estrangeira. Nossas considerações são de ordem prospectiva no sentido de que essa reflexão, para um sistema gerativo-transformacional do comportamento verbal, faz parte da atualidade e da evolução do ensino em termos de psicologia.

PALAVRAS-CHAVES: Comportamento verbal; Linguística das línguas estrangeiras; Pragmatismo.

\section{RÉFÉRENCES}

BACON, Francis. Nouvel Organum. In: BACON, Francis. Oeuvres de Bacon. 2. ed. Trad. Francis-Marie Riaux. Paris: Charpentier, 1851. p. 9-243.

BÉRARD, Evelyne. L’approche communicative : Théorie et pratiques. Paris: CLE International, 1991.

BERGSON, Henri. Matière et mémoire : Essai sur la relation du corps à l'esprit. 72. ed. Paris: Les Presses Universitaires de France, 1965.

CANALE, Michael ; SWAIN, Merrill. Theoretical Bases of Communicative Approaches to Second Language Teaching and Testing. Applied Linguistics, Oxford, vol. 1, n. 1, p. 1-47, 1980. Disponível em: < http://dx.doi.org/10.1093/applin/I.1.1>. Acesso em: 12 maio 2019. 
CHOMSKY, Noam. Un compte rendu du « comportement verbal» de R. F. Skinner. Langages, Paris, 4.16, p. 16-49, 1969. Disponível em: <www.persee.fr/doc/lgge 0458726x 1969 num 416 2016>. Acesso em: 19 fev. 2021.

CONSEIL DE L'EUROPE. Un cadre européen commun de référence pour les langues : apprendre, enseigner, évaluer. Paris: Didier, 2000.

DUMORTIER, Jean-Louis. Tâches-problèmes de communication et dispositif d'apprentissage en français langue seconde. In: VERDELHAN-BOURGADE, Michèle (Éd). Le français langue seconde : un concept et des pratiques en évolution. Bruxelles: De Boeck \& Larcier, 2007. p. 123-143.

HOAREAU, Saraly Huck; MÉRIEUX, Régine; LOISEAU, Yves. LATITUDES. 1 : Méthode de français. Guide pédagogique. Paris: Les Éditions Didier, 2008.

JAMES, William. Le pragmatisme (Éd.1911). Paris: Hachette Livre-BNF, 2018.

L'idée de vérité. Trad. Louis Veil e Maxime David. Paris: Librairie Félix Alcan, 1913.

MÉRIEUX, Régine; LOISEAU, Yves. LATITUDES. 1 : Méthode de français. Paris: Les Éditions Didier, 2008.

MOIRAND, Sophie. Enseigner à communiquer en langue étrangère. Paris: Hachette, 1982.

SKINNER, Frederic Burrhus. Verbal behavior. Englewood Cliffs, NJ: Prentice Hall, 1957.

Recebido em: 15/06/2021.

Aprovado em: 07/07/2021. 